\title{
Human pattern detection and recognition in the search for extraterrestrial intelligence
}

\author{
JOHN C. BAIRD \\ Dartmouth College, Hanover, New Hampshire 03755 \\ TYLER BLAKE \\ California State University, Northridge, California 91324 \\ and \\ TIMOTHY HEALY and JAMES SCHIMANDLE \\ Santa Clara University, Santa Clara, California 95053
}

\begin{abstract}
Potential radio signals from extraterrestrial civilizations will be weak with respect to background noise. We investigated people's ability $(N=18)$ to visually detect and recognize signals under noise conditions that simulate those expected to be present in an actual search. Linear signals were detected and identified better than pulses and wavy lines. In addition, observers sometimes agreed on the location of pseudosignals within a visual noise display (no actual signal present). Implications are noted for the search process as well as for advancing knowledge of the human visual system's capacity to detect and recognize signals.
\end{abstract}

It is anticipated that radio signals from extraterrestrial civilizations will be weak with respect to background noise, despite our possible success in choosing a suitable portion of the electromagnetic spectrum on which to focus a search (Cocconi \& Morrison, 1959; Drake, 1979; Makovetskii, 1980; Morrison, 1979;Seeger, 1977). Assuming the presence of a signal, one then is faced with detecting, recognizing, and interpreting its meaning. Here we address the detection-recognition problem.

The electronic computer and the human observer are the only detection devices receiving serious consideration by NASA in this endeavor (Lord, Note 1), which is termed the "Search for Extraterrestrial Intelligence" (SETI). A good deal is understood about the pattern perception capabilities of both systems, but almost nothing can be said about performance in a situation in which the signal characteristics are totally unknown. For example, such cases cannot be addressed by the usual methods arising from the theory of signal detectability (e.g., Baird \& Noma, 1978; Green \& Swets, 1974). We have collected data on humans' ability to visually detect and recognize signals embedded in noise fields that simulate the conditions expected in an actual SETI program (Seeger, 1977). Similar displays were used

This work was sponsored by the NASA-Ames Research Center and the University of Santa Clara as part of a study program of the American Society of Engineering Education. The essentials of this research were presented at the 1980 meeting of the Human Factors Society. We thank Bernard Oliver for providing the Hewlett-Packard video equipment. Requests for reprints should be sent to John C. Baird, Department of Psychology, Dartmouth College, Hanover, New Hampshire 03755. for a different purpose by Swets, Green, Getty, and Swets (1978).

\section{METHOD}

\section{Observers}

The participants were volunteers, 14 members of an engineering study program and 4 other professionals at the NASA-Ames Research Center. Although most were familiar with the general nature of the issues under investigation, none had any knowledge of the number, type, or location of signals employed. Each observer was tested individually.

\section{Apparatus}

The display was designed to simulate a matrix of radio frequency spectrum by time, in which the power in each matrix cell was represented by a brightness level on a black-and-white video unit (designed for this purpose by Hewlett-Packard). Different noise fields were employed for each condition, with signals superimposed to give the impression of a pattern of brighter pixels. Each noise field was generated over $2^{7}$ levels of amplitude, approximating a Rayleigh distribution, which is the distribution of the amplitude of bandpass Gaussian noise. These values were squared to approximate an exponential distribution of pixel (cell) intensities. ${ }^{1}$ Average signal-noise ratios (SNRs) in logarithmic units were $.4, .6, .8,1.0,1.2$, and 1.4 , with three patterns employed at each SNR: straight line, wavy line, and dashed line (pulse). In addition, six noise fields were presented without signals.

The starting point $(\mathrm{z})$ of a signal was randomly selected from positions across the top of the screen and extended to the bottom of a 128 by 128 matrix. The linear signal was computed as $y=m x+z$, where $y$ is the frequency offset from the upper left corner (columns) and $\mathrm{x}$ is time in seconds (rows). The pulse signal was generated as $y=m x+z$, where gaps in the array were produced by setting the pixel intensity equal to 0 when $\mathrm{x}<[\mathrm{d}+\mathrm{k} \operatorname{int}(\mathrm{x} / \mathrm{k})]$, and $\mathrm{d}$ is pulse duration and $\mathrm{k}$ is pulse length. The wavy line was generated as $y=m x+z+a \sin (2 \pi b x)$, where $a$ is the modulating limit and $b$ is the modulating frequency. 
These "call signals" might be considered by some civilization transmitting messages (Lord, Note 1 ). The linear signal would result if the sender concentrated power at a single frequency (or narrow band) over a continuous period of time. Due perhaps to the Doppler shift, we would receive this signal as a linear pattern drifting across the frequency spectrum as a function of time (unless Doppler-correction methods were used at the source). That is, we assume the transmitter is on a planet orbiting its parent star at a speed and distance sufficiently large to effect a continuous change in the received frequency over time. Another possible transmission strategy would be to focus a substantial amount of energy into a stream of pulses at a fixed frequency that, because of Doppler shift, would be received as a linear array of dashes drifting across the display. Linear signals (continuous or pulsed) represent obvious possibilities; the nonobvious alternatives are, of course, infinite. The wavy pattern represents the case of a signal that varies sinusoidally.

\section{Procedure}

First, the 24 displays were presented in a random order for approximately $10 \mathrm{sec}$ each. This phase familiarized the observer with the range and type of displays. Next, the series was repeated and the observer gave confidence ratings on a scale from 1 to 10 concerning the presence of a signal anywhere in the display. The procedure was self-paced, and the display was changed automatically after the observer's estimate was recorded. In the third phase, the series was repeated, and a second set of confidence ratings was given. In addition, observers drew what they considered to be potential signals on a sheet of graph paper (representing the display screen), which was divided into 64 equal zones ( 8 by 8 matrix). The observers were asked to draw at least one potential target on each sheet, even if they considered it an unlikely guess.

\section{RESULTS AND DISCUSSION}

There were small differences between confidence ratings on the two trials (phases), so these data were combined in further analyses. The mean confidence ratings are given in Figure 1 (as a function of SNR and pattern type), where it can be seen that the average rating for the noise fields was approximately 2.5 . Using this value as a lower referent, linear patterns were clearly detected

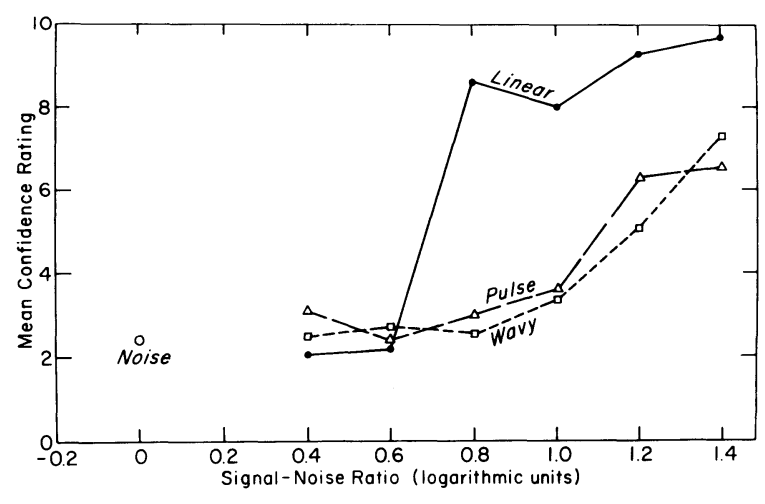

Figure 1. Mean confidence ratings $(N=36)$ of signal presence as a function of signal-noise ratio (SNR) in logarithmic units, where SNR values are measured as the ratio of signal intensity to the average intensity of background noise throughout the display. Data are based on judgments along a 10-point scale for each of three signal types (linear, pulse, wavy). at an SNR of .8, and pulses and wavy lines were detected at a higher value (1.0). Hence, by this standard, the absolute detection threshold was lowest for straight lines. Furthermore, once a straight line was detected, its presence was obvious, as is indicated by the sharp increase in confidence at an SNR near .8. On the other hand, the confidence ratings for the wavy lines and pulses increased gradually and fell significantly short of the maximum of 10, even with an SNR of 1.4.

The standard deviations (not shown here) also depended on the signal pattern, ranging from .8 (linear, $\mathrm{SNR}=1.4$ ) to 3.2 (wavy, SNR = 1.2). In general, variability was greater for the pulses and wavy lines than for the straight lines. The standard deviations also changed with SNR; greater variability occurred for the higher SNR values, with the exception of the straight lines, for which variability dropped dramatically at higher SNRs. Examination of individual data suggests that observers often split into two distinct subgroups: One subgroup definitely saw the signal, and the other missed it completely. The underlying reason for this bimodal distribution is unknown. One possibility is that some observers squinted or changed their state of visual accommodation, thus improving recognition of the signals (cf. Harmon \& Julesz, 1973).

The signal drawing data were first analyzed by calculating the percentage of hits and false alarms for each stimulus pattern. A hit was defined as any mark drawn in a cell of the 8 by 8 matrix that, in fact, contained part of a signal. The percentage of hits was the total number of correctly marked cells for all 18 observers divided by the total signal cells. Since this measure by itself may or may not reflect accuracy (e.g., a perfect score would result if observers marked every cell of the matrix), it was necessary to compute the false alarms as well. The percentage of false alarms was defined as the total number of marked cells in which a signal was not present, divided by the total number of nonsignal cells.

The percentage of hits as a function of SNR for each of the signal types was very similar to the confidence ratings presented in Figure 1. The Pearson productmoment correlation between percentage of hits and confidence ratings (for 18 signal conditions) was .89. For each signal pattern, the false alarm rate was at or below the rate for noise alone $(\sim 15 \%-20 \%)$. The linear signal yielded the lowest false alarm rate. In brief, the hit rates were not generated artifactually because a high percentage of the matrix was marked independent of a signal presence.

Histograms were constructed to show the location of drawn signals aggregated for all observers. Figure 2 (top) gives the frequency of drawn marks for the linear pattern at the highest SNR. [The matrix size is larger in the figure than on the data sheet ( 8 by 8 ) to show a transition between cells.] It is clear that observers showed substantial agreement on the location of this signal. Statistical tests (chi square) indicated that four of the six 

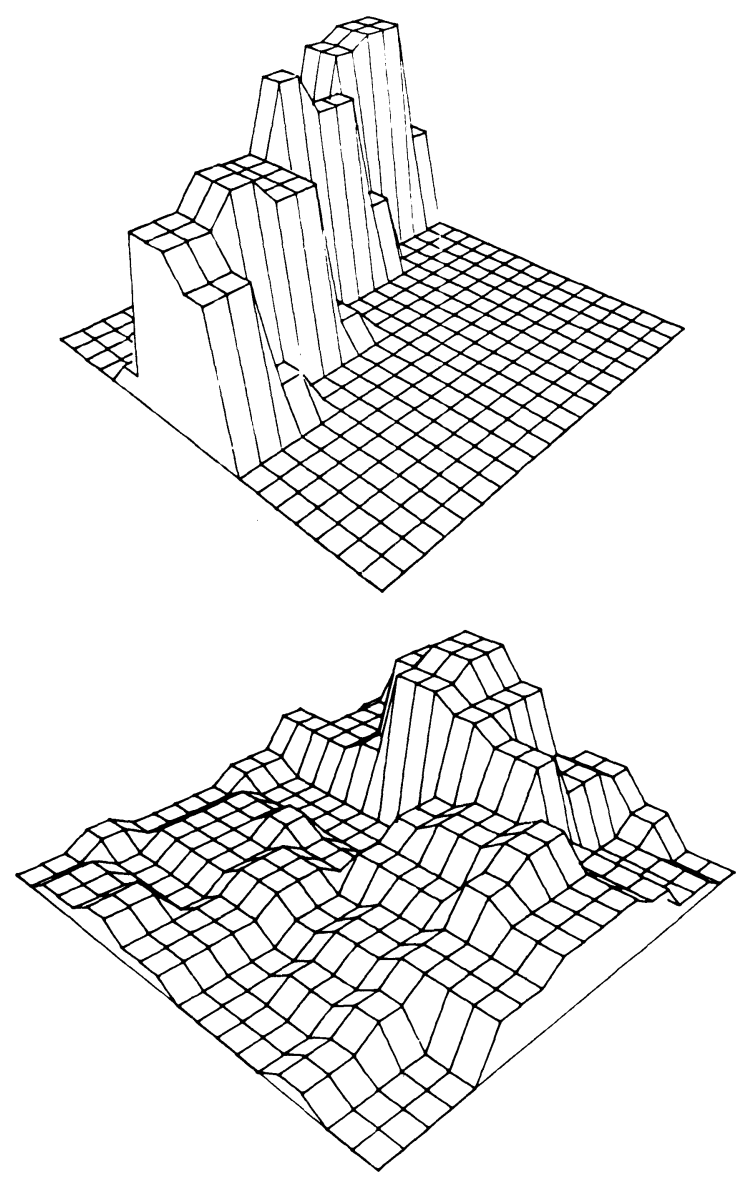

Figure 2. Frequency histogram of the location of drawings indicating recognition of a signal. Data based on drawings by 18 observers. Top: linear signal, $\mathrm{SNR}=1.4$. Bottom: noise field (no signal present).

histograms for the linear displays were significantly different from chance $(p<.05$, one-tailed test). With this same confidence level, two of the wavy-line histograms and two pulse histograms were significantly different from chance, as was one of the noise fields.

Figure 2 (bottom) presents rather surprising results for the significant noise field. The interesting aspect of this histogram is the agreement concerning the location of potential signals (although not significant, several other noise fields produced clear peaks and ridges). This outcome is intriguing because it suggests that characteristics of a noise display may lead people to infer signals, even though confidence in their presence is rather low (Figure 1). Detailed analyses of the stimulus characteristics leading to such false alarms may help us to better understand the feature detection capabilities of the human visual system (e.g., Fuld, 1978), although such biases may prove detrimental to a real-time search for actual signals from other civilizations. That is, we can expect a high percentage of false alarms to linear patterns that are generated by chance alone in the large fre- quency by time matrix being considered by NASA. [One plan under study is to have the human observer view small sections of an $8-\mathrm{MHz}$ by $1,000-\mathrm{sec}$ display (Lord, Note 1).]

Although limited in generality, the findings are provocative. Of particular interest for future research would be a comparison of automated and human detection performance on the same visual displays, including the analysis of stimulus characteristics associated with false alarms. The nature of the human-computer interaction in complex signal detection and recognition systems is not well understood. However, it is clear that the chief advantage of the human observer over the computer in the SETI context is that the exact nature of potential signals need not be specified beforehand. In order to program a computer to detect patterns, one must have a firm idea about the characteristics of the patterns to be detected. This is precisely the information we lack in our attempts to establish radio contact with extraterrestrial civilizations.

\section{REFERENCE NOTE}

1. Lord, S. (Ed.). Project OASIS: The design of a signal detector for the search for extraterrestrial intelligence (technical report). Santa Clara, Calif: University of Santa Clara, 1981.

\section{REFERENCES}

BaIrd, J. C., \& Noma, E. Fundamentals of scaling and psychophysics. New York: Wiley Interscience, 1978.

Cocconi, G., \& Morrison, P. Searching for interstellar communications. Nature, 1959, 184, 844-846.

Drake, F. D. A reminiscence of project Ozma. Cosmic Search, $1979,1,11-15$.

Fuld, K. A sensitization effect with rectilinear stimuli. Vision Research, 1978, 18, 1045-51.

GreEn, D. M., \& Swets, J. Signal detection theory and psychophysics (2nd ed.). Huntington, N.Y: Kreiger, 1974.

Harmon, L. D., \& Julesz, B. Masking in visual recognition: Effects of two dimensional filtered noise. Science, 1973, 180, 1194-1197.

MakovetskiI, P. V. Mutual strategy of search for CETI call signals. Icarus, 1980, 41, 178-192.

Morrison, P. Twenty years after . . . . Cosmic Search, 1979, 1, 7-9.

Segger, C. L. Search strategies. In P. Morrison, J. Billingham, \& J. Wolfe (Eds.), The search for extraterrestrial intelligence (NASA SP-419). Washington, D.C: U.S. Government Printing Office, 1977.

Swets, J. A., Green, D. M., Getty, D. J., \& Swets, J. B. Signal detection and identification at successive stages of observation. Perception \& Psychophysics, 1978, 23, 275-289.

\section{NOTE}

1. This was done because the light emitted by the CRT screen is approximately proportional to the amplitude of the beam. Hence, squaring the amplitudes leads to an image whose pixel intensities are proportional to power.

(Received for publication June 28, 1982.) 